\title{
Role of Phenotypic Switching in Stability and Persistence of Pseudomonas aeruginosa Biofilms
}

\author{
Zulfiqar Ali Mirani ${ }^{1}$, Shaista Urooj ${ }^{1,2}$, Fouzia Zeesahn ${ }^{3}$, Muhammad Naseem Khan ${ }^{1}$, Mubashir Aziz ${ }^{4}$, Izhar \\ Ahmed Shaikh ${ }^{5}$, Abdul Basit Khan ${ }^{1}$ \\ ${ }^{1}$ Microbiology Section-PCSIR Laboratories Complex Karachi, Pakistan \\ ${ }^{2}$ Department of Microbiology, Federal Urdu University of Arts, Science and Technology Karachi, Pakistan \\ ${ }^{3}$ Microbiology Dow University of Health Sciences, Karachi, Pakistan \\ ${ }^{4}$ Department of Pathobiology - Bahauddin Zakariya University, Multan, Pakistan \\ ${ }^{5}$ PCSIR Laboratories Complex Islamabad, Pakistan
}

\begin{abstract}
Objectives: Objective: This study was designed to explore the role of different phenotypes of $P$. aeruginosa in the development, stability and persistence of biofilm.

Methods: A total of seventeen (17) waterborne biofilm producing strains of $P$. aeruginosa were studied. These isolates were identified on the basis of typical phenotypic characters and the tube method was used for the study of biofilms. Population and phenotypic variance were studied by the drop plate method. The hydrophobicity of strains was evaluated by the bacterial adhesion to apolar solvent test.

Results: Study showed that the subject isolates of $P$. aeruginosa adopted a biofilm life style after $36 \mathrm{~h}$ of incubation at $35^{\circ} \mathrm{C}$. After $24 \mathrm{~h}$ the adhesion started, but it was reversible and easily dispersed by simple washing. However, after 36 $\mathrm{h}$ the irreversible adhesion was noticed. The biofilm consortia harbor three different phenotypes: i. wild types, showed typical $P$. aeruginosa characters on Cetrimide agar; ii. Slow growers, showed poor pigmentation and take $>36 \mathrm{~h}$ for colony development, and iii. Small colony variants (SCVs) are metabolically inactive and producing pinpointed non pigmented colonies. Comparative analysis showed that these phenotypes i.e. SCVs were highly hydrophobic and persistent in biofilm consortia due to the production of excessive amounts of exopolysaccharides.
\end{abstract}

Conclusions: This study showed that phenotypic heterogeneity is a characteristic feature of $P$. aeruginosa biofilms and all of these phenotypes have a major role in stability and persistence of biofilm consortia. $J$ Microbiol Infect Dis 2020; 10(1):10-17.

Keywords: Biofilms, Hydrophobicity, P. aeruginosa, Phenotypes, SCVs

$P$. aeruginosa is one of the versatile bacteria that evenly survive in clinical and community environs [1]. This ubiquitous Gram-negative bacterium is known as an opportunistic human pathogen, normally present in water, soil and on animate and inanimate objects [2]. Production of exopolysaccharides is a natural tendency of $P$. aeruginosa that enable these pathogens to adhere on animate and inanimate objects and protect them from the toxic effect of antibacterial agents [3], making $P$. aeruginosa an interesting and attractive model for biofilm study. Biofilms are bacterial communities connected and surrounded by extracellular matrix material and resistant to most of the available antibiotics and antiseptics [3]. It has been reported that most of the antibacterial agents are unable to penetrate in biofilm consortia, that leads to serious therapeutic problems in control of various infections [3,4]. Biofilms act a reservoir of 
pathogenic bacteria that can detach, resume their planktonic state, and contaminate new surfaces and patients [5]. $P$. aeruginosa is one of the leading colonizers in clinical setups including indwelling biomedical catheters and implants [1]. Most of the times these pathogens adopt a biofilm mode of life to nullify the toxic effect of antibiotics [3-5]. According to research reports, biofilm communities of $P$. aeruginosa consist of heterogeneous population, e.g. planktonic cells, sessile cells, persister or metabolically inactive population $[6,7]$. All of these phenotypes are different from each other in terms of growth rate, the pattern of gene expression, exopolysaccharides production, cell surface charges and antibiotic resistance $[6,7]$.

It has been reported that during the biofilm formation, bacteria display at least five distinct physiologies in order to survive and persist [8]. Similarly, different names have been reported in literature for highly adhesive and metabolically inactive colony variant phenotypes of $P$. aeruginosa e.g. small-colony variants (SCVs), rough small-colony variants (RSCVs), wrinkled variants, auto aggregating cells, and rugose colonies [6-8]. All of these metabolically inactive phenotypes are responsible for persistence or long term survival of $P$. aeruginosa in starvation, suffocation and in the presence of antibiotics or antiseptics [7-8]. According to Malone [9], the SCVs, small, auto-aggregative phenotypes of $P$. aeruginosa produce more exopolysaccharides and show the enhanced biofilm formation and strong attachment to surface. The biofilm formation and phenotypic heterogeneity are considered as an important aspect of $P$. aeruginosa pathogenicity, especially in chronic infections where they contribute in persistence and resistance to majority of antimicrobials [7-9]. The aim of present study is to explore the role of phenotypic heterogeneity in biofilm formation and stability.

\section{METHODS}

In the present study, a total of seventeen biofilm producing strains of $P$. aeruginosa were used. The subject strains were isolated from different water samples. These isolates were identified on the basis of typical morphology by Gram staining and growth on differential and selective media, e.g. Cetrimide Agar (Merck, Germany). The isolates were reconfirmed by the amplification of 16S rDNA of $P$. aeruginosa. The set of primers targeted species-specific signature sequences in 16S rDNA variable regions 2 and 8 (V2 and V8), respectively, using specific primers described by Spilker et al. [17].

\section{Biofilm Formation and Quantification}

A qualitative assessment of biofilm formation on glass slides was evaluated as described earlier by Mirani and Jamil [10]. Briefly, two-inch pieces of glass slides were submerged in $\mathrm{BHI}$ broth (Oxoid) containing $0.1 \mathrm{ml}$ of $4 \mathrm{hr}$ young culture of subject isolates of $P$. aeruginosa and were incubated at $35{ }^{\circ} \mathrm{C}$; results were analyzed after $24 \mathrm{~h}, 48 \mathrm{~h}, 72 \mathrm{~h}$ and $96 \mathrm{~h}$. After incubation, glass slides were washed with phosphate buffer saline ( $\mathrm{pH} 7.0)$ to remove unbound cells and debris. Biofilm formation was quantified as described previously by O'Toole et al. [11]. After washing with $0.85 \% \mathrm{NaCl}$ biofilms were fixed with acetic acid for $15 \mathrm{~min}$, stained with $0.3 \%$ crystal violet and rewashed. Biofilm-bound crystal violet was eluted in acetone: ethanol solution (1:4, by volume) and absorbance was recorded with Spectrophotometer (Nicollet Evolution 300 BB) at $563 \mathrm{~nm}$ wavelength.

\section{Enumeration of biofilm population}

After maturation of biofilm the glass slides (4 $\mathrm{mm}$ ) were gently washed three times with phosphate-buffered saline (PBS) to remove debris. After washing, glass slides were transferred to a sterile $5 \mathrm{ml}$ tube containing $3 \mathrm{ml}$ PBS and vortexed at $3000 \mathrm{rpm}$ for 2 minutes to separate cells from biofilms. After vortexing, the extracted bacteria were enumerated using the agar dilution plating technique. To perform it, 10 fold serial dilutions $(1 / 10,1 / 100$, and 1/1000) were made for each sample containing the dislodged bacteria and $10 \mathrm{ml}$ were seeded to calculate an accurate count of the biofilm population. Each experiment was performed in triplicate.

\section{Evaluation of colony variance during biofilm development and detection of persister cells}

The emergence of colony variants associated with biofilms of subject isolates was studied and these variants were enumerated, as described by Allegrucci and Sauer [12]. Biofilm biomass were harvested from a glass slide, resuspended in saline (total volume of $1 \mathrm{ml}$ ), homogenized for 30 s to disrupt cell clusters by vigorous shaking, serially diluted and plated on Tryptone Soy agar (Oxoid) and Cetrimide Agar (Oxoid). To determine stability of the colony variants, wellisolated colonies were sub-cultured on Tryptone Soy agar and incubated for $24 \mathrm{~h}$. This was 
repeated six times, and reversion with respect to colony size and biochemical reactions was monitored as described by Keren et al. [13]. The experiments were performed in duplicate. The drop plate method as described by Chen et al. [14] was followed to count CFUs.

\section{Scanning electron microscopy (SEM)}

Scanning electron microscopy was done to analyze the production of extracellular matrix material and cell morphology as described earlier [10]. Biofilm slides were divided into $4 \mathrm{~mm}$ sections and washed with distilled water to remove debris, and negatively stained with $0.2 \%$ uranyl acetate for $30 \mathrm{sec}$. These $4 \mathrm{~mm}$ slide sections showed the presence of biofilm material when examined directly in a GOELJEM-1200 EX II Electron Microscope.

\section{Bacterial hydrophobicity assay}

The hydrophobicity of strains was evaluated by the microbial adhesion to apolar solvent test as described by Kouidhi et al. [15]. It consisted of evaluating the affinity of the cells towards apolarsolvents (hexadecane). For the experiment, bacterial cells were harvested by centrifugation at $8500 \mathrm{~g}$ for $5 \mathrm{~min}$ and resuspended to Abs $578 \mathrm{~nm}$ in $0.01 \mathrm{M}$ potassium phosphate buffer (pH 7.0). This bacterial suspension was mixed with a solvent in a ratio of $1: 6(0.4 / 2.4 \mathrm{v} / \mathrm{v})$ by vortexing for $3 \mathrm{~min}$ to make an emulsion. The mixture was then left for 30 min until the separation of two phases. Aqueous phase absorbance was measured (Abs2) and the percentage of adhesion was expressed as: \% adhesion= $(1-\mathrm{Abs} 2 / \mathrm{Abs} 1) \times$ 100.

\section{Measurement of the polysaccharide in the supernatant of $P$. aeruginosa strains}

Quantification of exopolysaccharides was done as described previously by Qin et al. [16]. Briefly, $2 \mathrm{ml}$ of the cell free extract of subject isolates of $P$. aeruginosa was collected at different intervals, e.g. at 24h, 36h, 48h and $96 \mathrm{~h}$. A $1.0 \mathrm{ml}$ volume of $6 \%$ phenol was added, and allowed to react for $15 \mathrm{~min}$. Next, $2.5 \mathrm{ml} \mathrm{H}_{2} \mathrm{SO}_{4}$ was added to the solution, and allowed to react for $30 \mathrm{~min}$. The OD ${ }^{578}$ was then measured. TSB medium was used as a background control. The amount of polysaccharide in the supernatant of $P$. aeruginosa strains were calculated based on a standard glucose concentration curve, in which different concentrations of glucose were prepared in the same way as the $P$. aeruginosa supernatants.

\section{RESULTS}

In the present study, seventeen isolates of $P$. aeruginosa were used to study the in vitro biofilm formation process. These isolates were recovered from different water samples. It has been observed that the subject isolates adopted a biofilm life style after $36 \mathrm{~h}$ of incubation at 35 ${ }^{0} \mathrm{C}$. After $24 \mathrm{~h}$, the adhesion started, but it was reversible and easily dispersed by simple washing (Table 1). However, after $36 \mathrm{~h}$ the irreversible adhesion, difficult to disperse, was noticed. At this stage, the biofilm consortia harbor three different phenotypes i.e. wild types showed typical $P$. aeruginosa characters on cetrimide agar; slow growers showed poor pigmentation and take $>36 \mathrm{~h}$ for colony development; and the third phenotype has been metabolically inactive, very slow growing (usually appear after $>48 \mathrm{~h}$ of incubation) producing pinpointed transparent and nonpigmented colonies (Table 1).

This was also confirmed by scanning electron microscopy. Moreover, cells also exhibited different morphologies e.g. at log phase, the cells exhibited normal features of $P$. aeruginosa however, with the passage of incubation time when cells enter into biofilm stage or late stationary phase, they appear more elongated with cell wall being thickened (Figure $1 \mathrm{a}, \mathrm{b}, \mathrm{c}$ ). The small colony variants exhibit brick like appearance and rough surface with comparatively thin cell wall. Moreover, the cell wall of SCVs seems to be partially damaged.

Furthermore, scanning electron micrographs depicted that every individual cell in biofilm consortia was encased and interconnected with matrix material in an organized manner (Figure 1b, 1c). Interestingly, all of the subject isolates of $P$. aeruginosa exhibited similar biofilm architectures. Representative micrographs depicted that biofilms were composed of cells spread and interconnected like bacterial carpet, mainly composed of cell aggregates. The cell density and matrix material increases with incubation time and finally it appears like multicellular aggregates. Moreover, a highly adherent and phenotypically different variant 
looks like brick shape and embedded in matrix material was noticed in biofilm consortia.

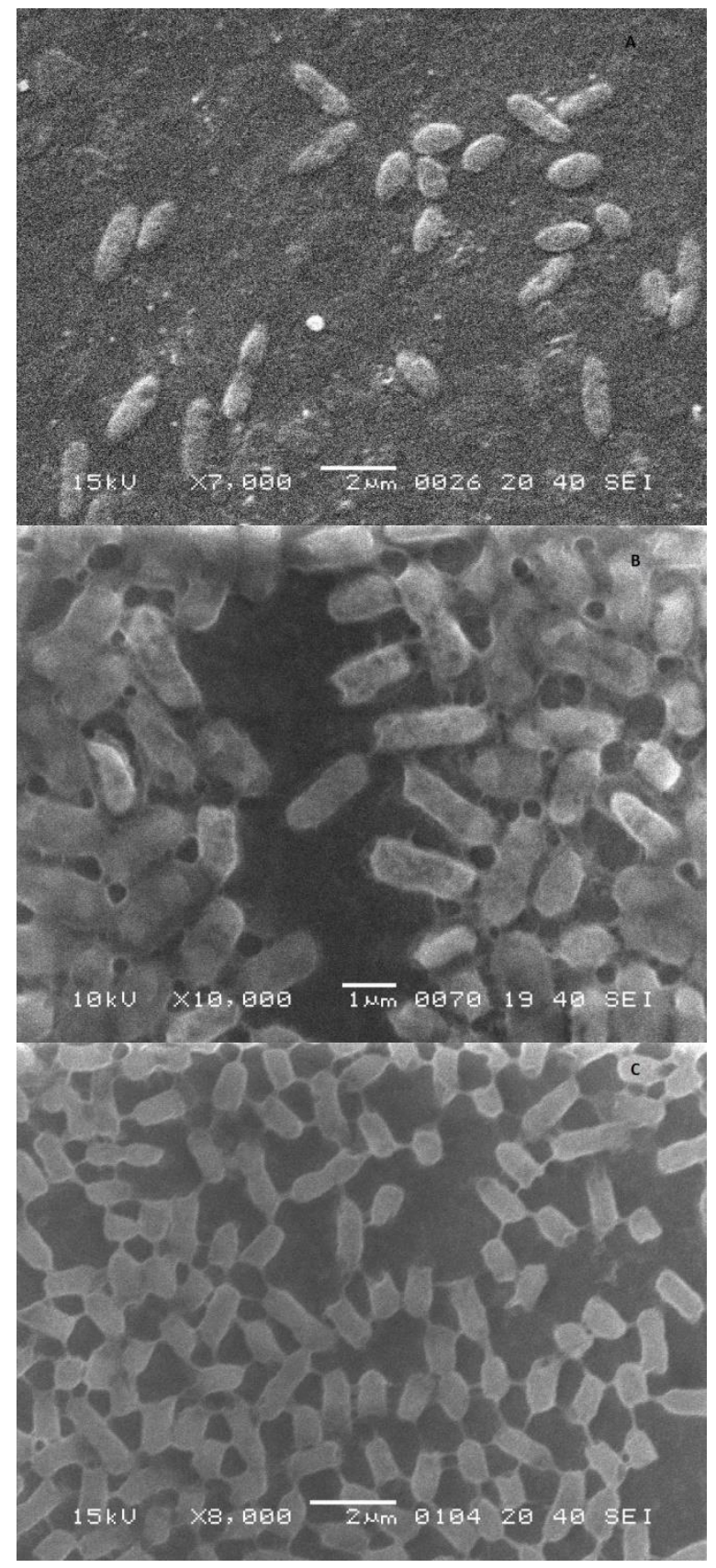

Figure 1: Scanning Electron Micrographs (A) Control (Planktonic stage) isolate of $P$. aeruginosa (B) Biofilm positive isolate depicted cells interconnected and covered with matrix material. (C) SCVs recovered from biofilm consortia after $48 \mathrm{~h}$ of incubation.

These phenotypes appeared after adaptation of biofilm life style i.e. after $36 \mathrm{~h}$ of incubation and increase with biofilm age and confirmed as SCVs by growth characters. Interestingly, increase of incubation time of biofilm consortia results in strong adhesion and dominance of SCVs (Table 1).

At $36 \mathrm{~h}$, out of seventeen ten isolates showed the presence of SCVs i.e. $>100 \mathrm{cfu} / \mathrm{ml}$ in biofilm consortia in others the SCVs count were $<10$ $\mathrm{cfu} / \mathrm{ml}$. After $48 \mathrm{~h}$, thirteen isolates showed the presence of SCVs (>100 cfu/ml) in biofilm consortia and the wild type population decreases (Table 1). After $96 \mathrm{~h}$, all of the isolates showed the presence of SCVs $(>100$ $\mathrm{cfu} / \mathrm{ml}$ ) in biofilm consortia. Furthermore, an increase in incubation time of biofilm consortia results in decrease in total population including wild type. Moreover, the optical OD also increases with the age of biofilm consortia. Further studies showed that biofilm $O D$ increases due to extracellular matrix material and was confirmed by the quantification of exopolysaccharides production (Figure 2).

The least quantity of exopolysaccharides recovered after $24 \mathrm{~h}$ and highest quantity was recovered after $96 \mathrm{~h}$ of incubation at $35{ }^{\circ} \mathrm{C}$. Scanning electron micrographs also supported these results (Figure 1b, 1c). Interestingly, the exopolysaccharides production and SCVs population of biofilm consortia increases, simultaneously. On pre-biofilm stage, the SCVs population was $<10 \mathrm{cfu} / \mathrm{ml}$ and recovery of exopolysaccharides was low. Furthermore, the hydrophobicity of biofilm consortia also correlates with SCVs, and exopolysaccharides production. The study showed that at the prebiofilm stage, the cells were comparatively hydrophilic; however, after adoption of a biofilm mode of life the cell surface hydrophobicity increases (Table 1). The highest level of hydrophobicity was noticed after $48 \mathrm{~h}$ of incubation when the consortia were dominated by SCVs.

\section{DISCUSSION}

$P$. aeruginosa is one of the most important bacterial pathogens responsible for community and hospital acquired infections [1]. It possesses intrinsic resistance to multiple antibiotics due to the presence of impermeable outer membrane and the presence of numerous multiple drug efflux pumps [1,2]. The other characteristic feature of $P$. aeruginosa that makes it more difficult to cure is the formation of biofilms. In biofilms, cells are encased in extracellular 
polymeric substances, e.g. polysaccharides, nucleic acids, lipids, proteins, etc. and are attached to each other and to the surface in a highly organized manner $[3,18]$.

The extracellular polymeric substances provide shelter to biofilm indwellers and protect them from toxic effect of antibiotics and other antibacterial agents [7]. Moreover, biofilm not only protect them from toxic materials, but also inhibit the supply of energy. It is reported that microbes growing in dense communities like biofilms can quickly exhaust their electron donors or acceptors and enter growth arrest due to energy limitations [19]. The present study showed that biofilm consortia of subject isolates of $P$. aeruginosa consist of heterogeneous population, i.e. wild type, slow growers as well as persister cells or SCVs. This population convergence from wild type to slow growing and SCVs increases with the age of biofilm and gradually dominated by metabolically inactive and highly adhesive SCVs. These phenotypes can survive for days to weeks and produce excessive amount of extracellular polymeric substances, even in the presence of very limited energy sources.

This study confirmed that the recovery of exopolysaccharides increases, even when the viable cell population begins to decline and it correlates with the population of SCVs in biofilm consortia. Although, SCVs have a major role in persistence and stability of biofilm, however, at initial stages these phenotypes were rarely found. This means that the initiative from planktonic to biofilm life style was attributed to wild type population. However, as biofilm progresses the SCVs population appears and dominates the consortia. This suggests that SCVs are the product of the biofilm environment that strengthen the adhesion through hydrophobic interaction and excessive production of extracellular polymeric substances.

This is also supported by previous studies [20, 21]. According to Häussler [20], the environment with limited nutrients favors the growth of SCVs and in the late stationary phase liquid cultures were found to have a selective advantage for the growth of these phenotypes. This is also supported by Onyango et al. [21]. They have reported that extreme environmental stresses such as prolonged exposure to low temperature, to very acidic or alkaline environments, or to osmotic stress may trigger SCV and/or persister cell formation. Moreover, scanning electron micrographs showed some morphological modification in subject isolates of $P$. aeruginosa.

On pre biofilm stage, the cells exhibited classical $P$. aeruginosa morphology. However, after adaptation of biofilm life style, the cells showed the elongated rod shape structure with rough surface. The surface roughness seems to be a characteristic feature of biofilm indwellers. It has been reported that cells with rough appearance are more hydrophobic and these phenotypes exhibit strong surface colonization and adhesion abilities [22]. Kaiser [23] described those bacteria which showed elongated cells, have the competitive advantage of the colony edge. In this regard, we hypothesize that morphological changes increase the surface area of the $P$. aeruginosa leading to a proper attachment to the surface and other cells.

Furthermore, the SCVs population of subject isolates of $P$. aeruginosa showed thin cells with rough surface and covered with extracellular matrix materials. The studies showed that SCVs are hydrophobic phenotypes with very low water content and are highly productive in extracellular matrix materials [24-25].

The size reduction or thinness may be attributed to low water content and surface roughness is due to increased production of extracellular matrix material. These qualities of SCVs are also responsible for hydrophobicity and enhanced adhesion to surface. Usually these phenotypes (SCVs) appear at the bottom of the biofilm due to reduced metabolic activity. Williamson et al., [26] described that $P$. aeruginosa biofilms harbor at least two distinct populations, the cells at the top are metabolically active and cells at the bottom are generally inactive with respect to expression of most genes or protein production. 
Table 1. Relationship of cell surface hydrophobicity, SCVs population and viable cell population in $P$. aeruginosa biofilms

\begin{tabular}{|c|c|c|c|c|c|c|c|c|c|c|c|c|c|c|}
\hline \multicolumn{4}{|c|}{$\begin{array}{c}\text { Biofilm Optical Density } \\
\text { (OD578a) }\end{array}$} & \multicolumn{4}{|c|}{ Cell Surface Hydrophobicity } & \multicolumn{4}{|c|}{ Viable Cell Population } & \multicolumn{3}{|c|}{$\begin{array}{c}\text { SCVs in Population of } \\
\text { Biofilms }\end{array}$} \\
\hline $24 \mathrm{~h}$ & $36 \mathrm{~h}$ & $48 \mathrm{~h}$ & $96 \mathrm{~h}$ & $24 \mathrm{~h}$ & $36 \mathrm{~h}$ & $48 \mathrm{~h}$ & $96 \mathrm{~h}$ & $24 \mathrm{~h}$ & $36 \mathrm{~h}$ & $48 \mathrm{~h}$ & $96 \mathrm{~h}$ & $36 \mathrm{~h}$ & $48 \mathrm{~h}$ & 96h \\
\hline 0.33 & 0.41 & 0.82 & 0.85 & 0.41 & 0.47 & 0.96 & 1.01 & $1 \times 105$ & $1 \times 105$ & $1 \times 104$ & $1 \times 102$ & $<10$ cfu & $1 \times 102$ & $1 \times 102$ \\
\hline 0.41 & 0.45 & 0.89 & 0.91 & 0.63 & 0.64 & 0.99 & 1.03 & $1 \times 105$ & $1 \times 104$ & $1 \times 103$ & $1 \times 102$ & $<10$ cfu & $1 \times 102$ & $1 \times 102$ \\
\hline 0.25 & 0.42 & 0.88 & 0.89 & 0.67 & 0.71 & 0.93 & 1.03 & $1 \times 105$ & $1 \times 105$ & $1 \times 103$ & $1 \times 102$ & $<10$ cfu & $1 \times 102$ & $1 \times 102$ \\
\hline 0.32 & 0.44 & 0.78 & 0.71 & 0.77 & 0.82 & 0.94 & 0.99 & $1 \times 105$ & $1 \times 105$ & $1 \times 103$ & $1 \times 103$ & $1 \times 101$ & $1 \times 101$ & $1 \times 102$ \\
\hline 0.24 & 0.44 & 0.99 & 0.98 & 0.72 & 0.82 & 0.92 & 1.01 & $1 \times 105$ & $1 \times 105$ & $1 \times 103$ & $1 \times 103$ & $1 \times 102$ & $1 \times 102$ & $1 \times 102$ \\
\hline 0.42 & 0.49 & 1.11 & 1.09 & 0.73 & 0.85 & 0.91 & 0.97 & $1 \times 105$ & $1 \times 104$ & $1 \times 103$ & $1 \times 102$ & $1 \times 102$ & $1 \times 102$ & $1 \times 102$ \\
\hline 0.36 & 0.47 & 0.91 & 0.99 & 0.67 & 0.79 & 0.89 & 0.99 & $1 \times 105$ & $1 \times 104$ & $1 \times 103$ & $1 \times 102$ & $1 \times 102$ & $1 \times 102$ & $1 \times 102$ \\
\hline 0.33 & 0.44 & 0.52 & 0.99 & 0.59 & 0.79 & 0.68 & 0.97 & $1 \times 105$ & $1 \times 105$ & $1 \times 103$ & $1 \times 102$ & $<10 \mathrm{cfu}$ & $<10 \mathrm{cfu}$ & $1 \times 102$ \\
\hline 0.39 & 0.51 & 0.88 & 0.97 & 0.62 & 0.69 & 0.90 & 1.04 & $1 \times 105$ & $1 \times 105$ & $1 \times 103$ & $1 \times 102$ & $1 \times 102$ & $1 \times 102$ & $1 \times 102$ \\
\hline 0.26 & 0.46 & 0.88 & 0.95 & 0.74 & 0.77 & 0.92 & 1.05 & $1 \times 105$ & $1 \times 104$ & $1 \times 103$ & $1 \times 103$ & $1 \times 101$ & $1 \times 102$ & $1 \times 102$ \\
\hline 0.34 & 0.47 & 0.56 & 0.96 & 0.77 & 0.82 & 0.85 & 0.97 & $1 \times 105$ & $1 \times 104$ & $1 \times 103$ & $1 \times 103$ & $1 \times 101$ & $1 \times 102$ & $1 \times 102$ \\
\hline 0.27 & 0.39 & 0.79 & 0.98 & 0.72 & 0.77 & 0.87 & 0.99 & $1 \times 105$ & $1 \times 105$ & $1 \times 103$ & $1 \times 102$ & $<10$ cfu & $<10$ cfu & $1 \times 102$ \\
\hline 0.33 & 0.36 & 0.97 & 1.13 & 0.64 & 0.71 & 0.84 & 0.98 & $1 \times 105$ & $1 \times 104$ & $1 \times 103$ & $1 \times 103$ & $1 \times 102$ & $1 \times 102$ & $1 \times 102$ \\
\hline 0.43 & 0.52 & 0.98 & 1.13 & 0.68 & 0.72 & 0.83 & 0.98 & $1 \times 105$ & $1 \times 104$ & $1 \times 103$ & $1 \times 103$ & $1 \times 102$ & $1 \times 102$ & $1 \times 102$ \\
\hline 0.41 & 0.59 & 0.95 & 1.12 & 0.66 & 0.72 & 0.94 & 1.06 & $1 \times 105$ & $1 \times 105$ & $1 \times 103$ & $1 \times 103$ & $<10$ cfu & $<10$ cfu & $<10 \mathrm{cfu}$ \\
\hline 0.32 & 0.59 & 0.62 & 0.99 & 0.49 & 0.63 & 0.76 & 1.01 & $1 \times 105$ & $1 \times 105$ & $1 \times 103$ & $1 \times 102$ & $<10$ cfu & $<10$ cfu & $1 \times 102$ \\
\hline 0.33 & 0.55 & 0.61 & 0.88 & 0.43 & 0.55 & 0.73 & 0.98 & $1 \times 105$ & $1 \times 105$ & $1 \times 103$ & $1 \times 102$ & $1 \times 101$ & $1 \times 102$ & $1 \times 102$ \\
\hline
\end{tabular}

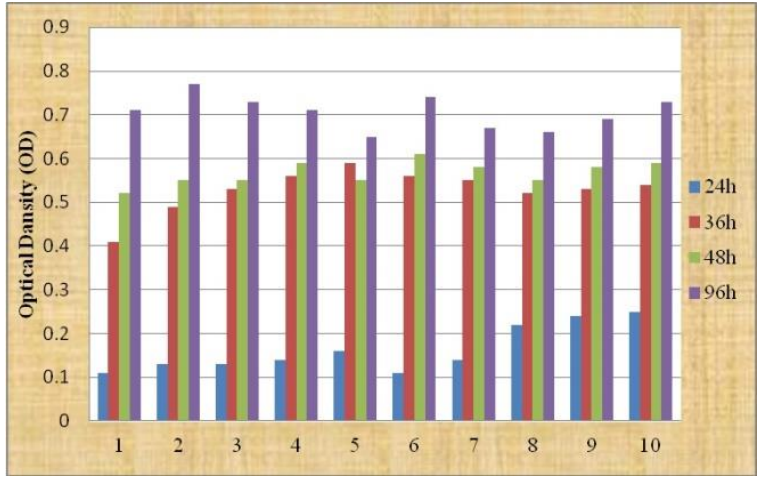

Figure 2. Exopolysaccharides production by biofilm producer isolates of $P$. aeruginosa.

All of these changes in the life style of $P$. aeruginosa have a major contribution in pathogenesis and survival of $P$. aeruginosa. Planktonic cells are more active, aggressive and equipped for host invasion.

However, these phenotypes are also vulnerable to host defense and other antibacterial agents. Contrary to these, the cells in biofilms are slow growing metabolically inactive, and highly resistant to antibacterial agents, host defenses and persist for a long time. This suggests that planktonic cells are responsible for acute infections while biofilm are contributing in chronic infections. This is in agreement with Valentini et al. [27] and Moradali et al. [7]. They have described that formation of mucoid biofilm by $P$. aeruginosa is the hallmark of chronic infections and indicative of disease progression and long-term persistence. Interestingly, this phenotypic switch appears with special task and time. Bacterial community composed of functionally diverse populations is likely to perform better in general because of the likelihood that some subpopulation will thrive as prevailing conditions change. Wild type initiates the process of infection and utilizes available energy sources. During the process the metabolic activities of cells are promoted by nutrients and oxygen rich environment in the periphery biofilm, which supports bacteria in proliferation [28]. As the biofilm consortia progresses, the cells are affected by the depletion of energy and oxygen sources due to poor diffusion. This limits the metabolic potential inside the niche and results in reduction of growth rate and development of starved cells in the biofilm consortium [29]. It is a well-known phenomenon that bacteria with reduced metabolism and growth rate are less susceptible to the antimicrobial agents [28-30]. In the process, the next stage is the development of persister cells or small colony variants. These dormant phenotypes are responsible for the long term survival of $P$. aeruginosa in highly stressful environment. They are highly hydrophobic, 
adhesive and producing excessive amounts of extracellular matrix material to connect and protect the biofilm indwellers under stress environment.

This is an in vitro study based on single species model. The conditions and environment generated to study the phenotypic behavior of $P$. aeruginosa in stress environment. In natural environment in the presence of multiple partners bacteria may respond differently.

\section{Conclusion}

Phenotypic heterogeneity is a characteristic feature of $P$. aeruginosa biofilms. The wild type phenotypes have a major role in the initiation of infection processes, while slow growing phenotypes and SCVs have an important role in the stability and persistence of biofilm consortia.

\section{ACKNOWLEDGMENTS}

We are thankful to Mr. M Yousf Khan of University of Karachi for providing Scanning Electron Microscopy and Sindh Institute of Urology and transplantation Transmission Electron Microscopy facilities for this study.

Competing interests: The authors declare no competing interest.

Funding: The authors received no funding for this study.

All authors of this research paper have directly participated in the planning, execution, or analysis of this study.

\section{REFERENCES}

1. Sousa AM, Pereira MO. Pseudomonas aeruginosa Diversification during Infection Development in Cystic Fibrosis Lungs-A Review. Pathogens (Basel, Switzerland) 2014; 3(3):680703.

2. Sadikot RT, Blackwell TS, Christman JW, Prince AS. Pathogen-host interactions in Pseudomonas aeruginosa pneumonia. Am J Respir Crit Care Med 2005; 171: 1209-1223.

3. Algburi A, Comito N, Kashtanov D, Dicks LM, Chikindas ML. Control of biofilm formation: Antibiotics and beyond. Appl Environ Microbiol 2017; 83. doi:10.1128/AEM.02508-16.

4. Alguri A, Comito N, Kashtanov D, Dicks LM, Chikindas ML. Control of Biofilm formation:
Antibiotics and beyond. Appl Environ Microbio Mo IBiol Rev 2014: 78:510-543.

5. Machado I, Graça J, Lopes H, Lopes S, Pereira MO. Antimicrobial pressure of ciprofloxacin and gentamicin on biofilm development by an endoscope-isolated Pseudomonas aeruginosa. ISRN Biotechnol 2012; 2013: 178646.

6. Hall CW, Mah TF. Molecular mechanisms of biofilm-based antibiotic resistance and tolerance in pathogenic bacteria. FEMS Microbiol Rev 2017; 41: 276-301. doi: 10.1093/femsre/fux010.

7. Moradali MF, Ghods S, Rehm BHA. Pseudomonas aeruginosa lifestyle: A paradigm for adaptation, survival, and persistence. Front. Cell. Infect. Microbiol 2017; 7-39.

8. Sauer K, and Camper AK. Characterization of phenotypic changes in Pseudomonas putida in response to surface-associated growth. J Bacteriol 2001; 183: 6579-6589.

9. Malone JG. Role of small colony variants in persistence of Pseudomonas aeruginosa infections in cystic fibrosis lungs. Infect Drug Resist 2015; 8: 237-247.

10. Mirani ZA, Jamil N. Effect of sub-lethal doses of vancomycin and oxacillin on biofilm formation by vancomycin intermediate resistant Staphylococcus aureus. J Basic Microbiol 2011; 51: 191-195.

11. O'Toole GA, Kolter R. Initiation of biofilm formation in Pseudomonas fluorescens WCS365 proceeds via multiple, convergent signalling pathways: a genetic analysis. Mol Microbiol 1998; 28: 449-461.

12. Allegrucci M, Sauer K. Characterization of colony morphology variants isolated from Streptococcus pneumonia biofilms. J Bacteriol 2007; 189: 20302038.

13. Keren I, Kaldalu N, Spoering A, Wang Y, Lewis K. Persister cells and tolerance to antimicrobials. FEMS Microbiol Lett 2004; 230:13-18.

14. Chen CY, Nace GW, Irwin PL. A $6 \times 6$ drop plate method for simultaneous colony counting and MPN enumeration of Campylobacter jejuni, Listeria monocytogenes, and Escherichia coli. J Microbiol Meth 2003; 55: 475-479.

15. Kouidhi B, Zmantar T, Hentati H, Bakhrouf A. Cell surface hydrophobicity, biofilm formation, adhesives properties and molecular detection of adhesins genes in Staphylococcus aureus associated to dental caries. Microb Pathog 2010; $49(1-2): 14-22$.

16. Qin Z, Yang Y, Qu D, Molin S, Tolker-Nielsen T. Pseudomonas aeruginosa extracellular products inhibit staphylococcal growth, and disrupt established biofilms produced by Staphylococcus epidermidis. Microbiol 2009; 155: 2148-2156.

17. Spilker T, Coenye T, Vandamme P, LiPuma JJ. PCR-based assay for differentiation of 
Pseudomonas aeruginosa from other Pseudomonas species recovered from cystic fibrosis patients. J ClinMicrobiol 2004; 42: 20742079.

18. Ma L, Conover M, Lu H, Parsek MR, Bayles K, Wozniak DJ. Assembly and development of the Pseudomonas aeruginosa biofilm matrix. PLoS Pathog 2009; 5:e1000354.

19. Basta DW, Bergkessel M, Newman DK. Identification of fitness determinants during energy-limited growth arrest in Pseudomonas aeruginosa. mBio 2017; 8:e01170-17.

20. Häussler S, Ziegler I, Löttel A, et al. Highly adherent small-colony variants of Pseudomonas aeruginosa in cystic fibrosis lung infection. $\mathrm{J}$ Med Microbiol 2003; 52:295-301.

21. Onyango LA, Hugh Dunstan R, Roberts TK, Macdonald MM, Gottfries J. Phenotypic variants of staphylococci and their underlying population distributions following exposure to stress. PLoS One 2013; 8:e77614.

22. Petráčková $D$, Buriánková $K$, Tesařová $E$, et al. Surface hydrophobicity and roughness influences the morphology and biochemistry of streptomycetes during attached growth and differentiation. FEMS Microbiol Lett 2013; 342, 147-156.

23. Kaiser D. Bacterial swarming: a re-examination of cell-movement patterns. Curr Biol 2007; 17: R561-R570.

24. Johns BE, PurdyKJ, Tucker NP, Maddocks SE. Phenotypic and genotypic characteristics of small colony variants and their role in chronic infection. Microbiol Insights 2015; 8: 15-23.

25. Sandt C, Smith-Palmer T, Comeau J, Pink D. Quantification of water and biomass in small colony variant PAO1 biofilms by confocal Raman microspectroscopy. Appl Microbiol Biotechnol 2009; 83 (6): 1171-1182.

26. Williamson KS, Richards LA, Perez-Osorio AC, et al. Heterogeneity in Pseudomonas aeruginosa biofilms includes expression of ribosome hibernation factors in the antibiotic-tolerant subpopulation and hypoxia-induced stress response in the metabolically active population. $J$ Bacteriol 2012; 194: 2062-2073.

27. Valentini M, Gonzalez D, Mavridou DA, Filloux A. Lifestyle transitions and adaptive pathogenesis of Pseudomonas aeruginosa. Curr Opin Microbiol 2017; 18:41:15-20.

28. Singh S, Singh SK, Chowdhury I, Singh R. Understanding the Mechanism of Bacterial Biofilms Resistance to Antimicrobial Agents. The open microbiology journal. 2017; 11:53-62.

29. Wentland EJ, Stewart PS, Huang CT, McFeters GA. Spatial variations in growth rate within
Klebsiella pneumoniae colonies and biofilm. Biotechnol Prog 1996; 12(3):316-321.

30. Boles BR, Thoendel M, Singh PK. Self-generated diversity produces "insurance effects" in biofilms communities. Proc Natl Acad Sci USA 2004; 101: 16630-16635. 